\title{
Ein neues Lot zur Untersuchung der obersten Schlammschichten, zur Messung des Sedimentabsatzes und zur Erfassung bodennaher Wasserschichten
}

\author{
Von H. ZüLLIG, Rheineck
}

(Mitteilung aus der Eidgenössischen Anstalt für Wasserversorgung, Abwasserreinigung und Gewässerschutz an der Eidgenössischen Technischen Hochschule, Zürich; Direktion: Prof. Dr. O. JAAG)

Eingegangen am 26. Oktober 1953

Die exakte Erfassung eines Gewässerzustandes gehört in den engsten Problemkreis der experimentellen Limnologie. Intensive Forschungen auf den Gebieten der Wasserbiologie und Wasserchemie führten in den letzten Jahrzehnten zu Erkenntnissen, auf Grund derer es heute weitgehend möglich ist, den Trophiegrad eines stehenden oder fliessenden Gewässers pauschal zu erfassen. Die Charakterisierung gelingt dabei um so exakter, je häufiger Probenahmen durchgeführt werden können.

Untersuchungen an Gewässersedimenten verschiedensten Trophiegrades zeigten, dass die Schlammablagerungen bei sinnvoller Analysierung weitgehend Rückschlüsse auf den Verschmutzungsgrad der überstehenden Wassermassen ziehen lassen (O. JAAG und H. ZüLLIG [4] $]^{\wedge}$ ).

Nebst der eigentlichen Analyse bildet die einwandfreie Probenahme die Grundlage einer zweckmässigen Analysierung. Der Übergang von der tiefsten Schicht des freien Wassers zum Seeboden erfolgt meist kontinuierlich, indem die allerobersten Schlammschichten eine Art Schlammwasserphase bilden, deren Gehalt an Wasser oft $95-99 \%$ beträgt. Die Festbestandteile dürften vorwiegend aus hydratisierten Kolloiden bestehen. Bereits in $5-10 \mathrm{~cm}$ Tiefe nimmt dann der Wassergehalt merklich ab.

1) Die Ziffern in eckigen Klammern verweisen auf das Literaturverzeichnis auf Seite 285. 
Untersuchungen von OHLe [8], Alsterberg [I], Mortimer [6], HutCHINSON [3] und andern Autoren zeigten, dass sich zwischen Schlamm und Wasser Austauschvorgänge abspielen, deren Grösse von verschiedensten Faktoren abhängig ist. Perviliev [9] und LIEBMann [ 5 ] weisen auf die biologische Mikroschichtung an den Kontaktzonen WasserSchlammwasser-Schlamm hin, in denen sich die Hauptabbauprozesse sedimentierten Materials abspielen.

Im Rahmen unserer Schlammuntersuchungen schien es uns daher erwünscht, ein besonderes Augenmerk auf Chemie und Biologie dieser Kontaktzonen zu richten und Methoden zu entwickeln, welche auf einfache Weise gestatten, rasch das gewünschte Material zu erhalten.

Durch die Arbeiten Hampus von Post (I 862 [Io]) und PotonIÉ (I908 [II]) begannen sich die Limnologen für die Gewässerablagerungen in den Binnengewässern zu interessieren. NAUMANN führte 1917 [7] die ersten Becher- und Rohrlote in der hydrobiologischen Untersuchungsmethodik ein. In diesen Jahren entwickelten EKMAN, BIRGE und ALSTERBERG Bodengreifer, welche in spätern Jahren - ebenso wie das Naumann-Lot verschiedentlich modifiziert wurden.

Während die Bodengreifer die Schlammschichtung weitgehend stören und ausschliesslich Schlamm zu heben vermögen, liegt ihr Vorteil darin, dass sie die Möglichkeit bieten, grössere Schlammengen der faunistischen und chemischen Untersuchung zugänglich zu machen. Durch die Schlammentnahme mittels des Naumann-Lotes, dessen Rohreinsatz zirka $25 \mathrm{~mm}$ Innendurchmesser aufweist, gelingt es indessen nur, kleine, jedoch ungestörte Schlammbohrkerne zu heben. Die Untersuchung solcher Bohrkerne gestaltet sich insofern schwierig, als beim Ausstossen derselben die allerobersten Schichten, oft die interessantesten, im allgemeinen infolge ihres hohen Wassergehaltes zerstört werden und der Analyse entgehen. Ausserdem zirkuliert beim Versenken des mit Deckelventil versehenen Naumann-Lotes das Wasser nicht genügend in der Glasröhre, so dass die in der Röhre den Bohrkern überlagernde Wassersäule nicht sicher dem bodennahen Wasser des Sees entspricht. Sowohl Schlamm- als auch Wassermenge genügen bei der Probenahme mittels des kleinen NaumannLotes nicht, um chemische Untersuchungen durchzuführen. Diese Gründe mochten auch NAUMANN bewogen haben, den kombinierten WasserBodenschöpfer von $5 \mathrm{~cm}$ Rohrdurchmesser zu konstruieren, an welchem das bodennahe Wasser durch seitlich angebrachte Hähne abgezapft werden konnte. Die Ausführung in Metall gestattete ihm vermutlich nicht, die Grenze zwischen Schlamm und Wasser genau festzustellen. Ob ferner 
in diesem kombinierten Apparat die einwandfreie Zirkulation gesichert war, entgeht unserer Kenntnis.

Im Jahre 1948 begann der Verfasser mit der Konstruktion von für unsere Zwecke idealeren Rohrloten, welche an Stelle der Deckelventile Klappventile besassen und durch ein Fallgewicht erst nach dem Einstich in den Seeboden geschlossen werden konnten. Als Einsatzröhren wurden Plexiglasröhren verwendet, welche sich leicht bearbeiten lassen. Offenbar gleichzeitig arbeitete LIEBMANN [s] an der Konstruktion eines verbesserten Rohrlotes zur Untersuchung der Mikroschichtung. Er verwendete ein Messingrohr, welches im Abstand von I mm Bohrungen von $0,5 \mathrm{~mm}$ Durchmesser trug, die mittels Nitrolack gedichtet wurden. Zur Untersuchung der Mikroschichtung führt LIEBMANN Pipetten durch die lakkierten Bohrungen ein. Als Ventil verwendet er ebenfalls ein Klappventil, welches jedoch am untern Ende der Röhre angebracht ist und durch eine Leine betätigt wird.

Unsere Erfahrungen im Gelände führten uns schliesslich zur Konstruktion der in Abbildung I dargestellten Bohreinrichtung. Eine $\mathrm{I}, 3 \mathrm{~m}$ lange Plexiglasröhre von $40 \mathrm{~mm}$ äusserem Durchmesser und $3 \mathrm{~mm}$ Wandstärke wird mittels Flügelmuttern in ein mit Stabilisierungsflügeln versehenes, zirka $5 \mathrm{~kg}$ schweres Rohr eingepresst, welches oben ein Deckelventil enthält. Letzteres wird durch zwei Haltevorrichtungen beim Versenken offengehalten, womit man eine einwandfreie Wasserzirkulation erzielt. An der Peripherie der Stabilisierungsflügel sind Führungen angebracht, welche den sogenannten «Fühler» führen. Stösst der Ring des «Fühlers» nach Einstich des Rohres in den Schlamm auf, so bewegt sich das Gestänge nach oben und klinkt die Ventilhaltevorrichtung aus, so dass sich das Ventil während des Einstichvorganges frei bewegen und sofort schliessen kann. Diese automatische Ventilschliessvorrichtung weist den Vorteil auf, dass der Schlammstecher bei hohem Wellengang nicht bereits wieder aus dem Boden gerissen wird, bevor ein Fallgewicht das Ventil geschlossen hat. Die Fühlervorrichtung verschiebt ausserdem den Schwerpunkt des Lotsystems nach unten und wirkt daher stabilisierend.

Bearbeitete Plexiglasröhren sind meistens in ihrer Biegefestigkeit geschwächt. In sandigen Sedimenten kam es nicht selten vor, dass Plexiglasröhren brachen. Aus diesem Grunde haben wir eine Metallschutzröhre, welche den bearbeiteten Teil der Plexiglasröhre deckt, entwickelt, welche dem Glasrohr eng anliegt und unten einen abschraubbaren Schneidefuss enthält. Aussen- und Innendurchmesser dieses Fusses sind so dimensioniert, dass die Sedimentsäule nur unten ausgestochen und mit kleinerer 
Mantelreibung gefasst werden kann. Das Zusammenstauchen der Bohrkerne, insbesondere der obersten $20 \mathrm{~cm}$, welche noch wasserreich sind, wird dadurch vermindert.

Die Plexiglasröhre (Abb. 2) enthält in verschiedenen Abständen, meist

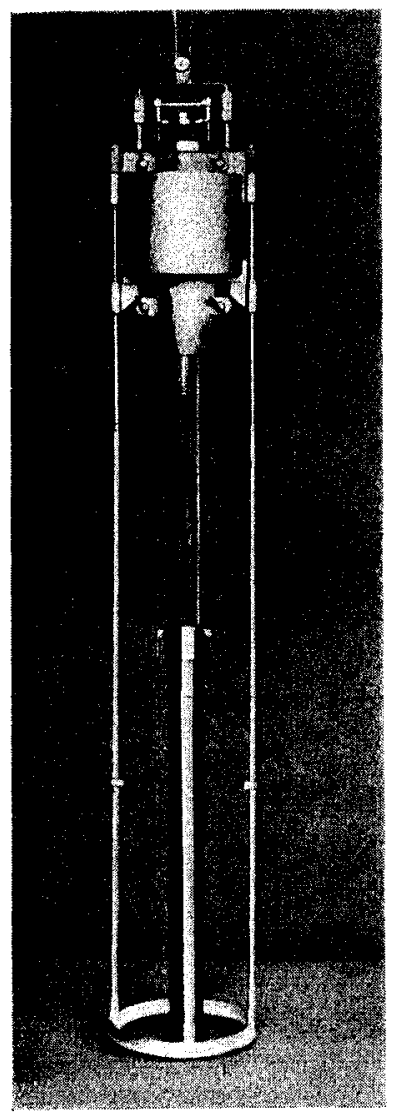

Abb. 1

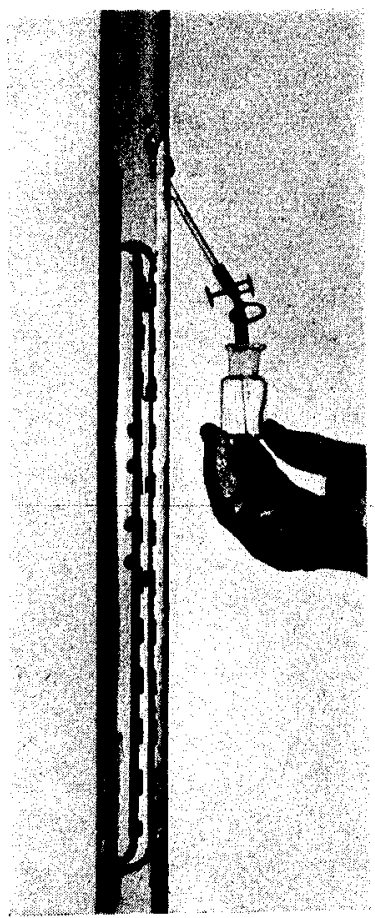

Abb. 2

von $20 \mathrm{zu} 20 \mathrm{~cm}$, Bohrungen von $5 \mathrm{~mm}$ Durchmesser, welche mit Weichgummischeibchen von $8 \mathrm{~mm}$ Durchmesser, ebenfalls in die Röhre eingelassen, gedichtet werden. Aus diesen Bohrungen können mittels einer Injektionsnadel, welche als Verlängerung einen Gummischlauch mit Quetschhahn aufweist, Proben zu Mikro-Sauerstoffbestimmungen und weitern mikrochemischen Untersucbungen entnommen werden. Die Gummischeibchen las- 
sen sich leicht von der Nadel durchstossen und dichten beim Herausziehen sofort ab. Die für die Aufnahme des Bohrkerns gedachte untere Rohrpartie enthält ebenfalls, jedoch im Abstande von nur I cm, gegenseitig versetzt, gummigedichtete Bohrungen. Dadurch wird die Entnahme interessanter Mikroschichten für die mikroskopiscbe Analyse und zu bakteriologischen Untersucbungen ermöglicht.

Schliesslich dient die Röhre der quantitativen Messung des Scblammabsatzes. Die im folgenden beschriebene Methode nennen wir die "photographische Schichtenbezugmethode». Sie wurde insbesondere während der Schlammuntersuchungen im Luganersee entwickelt. An einer möglichst genau einvisierten Stelle fasst man eine Schlammprobe und schneidet sie in der Plexiglasröhre mittels einer $0,5 \mathrm{~mm}$ dicken Plexiglasscheibe der ganzen Länge nach durch. Hierauf öffnet man das in die Röhre eingeschnittene Fensterchen, wischt den vor dem Glas sich befindenden Schlamm weg und photographiert den Plexiglasstreifen mit eingeritzter Millimeterskala und den dahinter sich befindenden Schlamm, dessen Schichtung auf diese Weise sehr deutlich hervortritt. Der so präparierte Schlamm wird nun zur spätern Dokumentation photographiert. Für diese $Z$ wecke haben sich Plattenapparate am besten bewährt. Auf der Mattscheibe des Photoapparates wird einmal die genaue Distanz Linse-Objekt eingestellt und nachher an Stelle der Mattscheibe ein Filmpack eingeschoben. Aufnahmeapparat und Röhre sind miteinander durch ein horizontales Stativ starr verbunden (Abb. 3). Auf diese Weise gelingt es, selbst bei starkem Wellengang unverzerrte Bilder zu gewinnen.

In gewissen Zeitabständen werden an der gleichen Stelle gestochene Bohrkerne gleichermassen aufgeschnitten und photographiert. Die meisten Sedimente weisen für das Auge herausstechende Schichten auf, die oft durch Einschwemmungen entstanden und anders als das übrige Sedimentmaterial gefärbt sind. Solche Schichten verwenden wir, falls nicht bereits typische Jahresschichtung vorhanden ist, als Bezugsschichten, von denen an der jahreszeitliche Zuwachs an Sedimentmaterial mittels der Millimeterskala verfolgt werden kann. Zu diesem Zweck legt man die photographischen Aufnahmen nebeneinander.

Die Schichthöhenangabe erfordert auch die Angabe des Wassergehaltes, da ältere Schichten durch neu anfallendes Sedimentmaterial zusammengepresst werden und an Wasser verlieren. Zur Wassergehaltsbestimmung. füllt man Sedimentmaterial mittels eines feinen Spatels nach dem Aufschneiden und Photographieren in möglichst kleine, eingewogene Probegläschen und verschliesst dicht mit Gummistopfen. Im Laboratorium 
trocknet man die Gläschen mit Inhalt bei $80^{\circ} \mathrm{C}$ während $4 \mathrm{~h}$, lässt im Trockenschrank erkalten und wägt. Der Wassergehalt ergibt sich aus der Gewichtsdifferenz.

Die Schichtenbezugmethode lässt sich indessen nicht überall zur Messung des Sedimentabsatzes anwenden. Insbesondere versagt sie in der

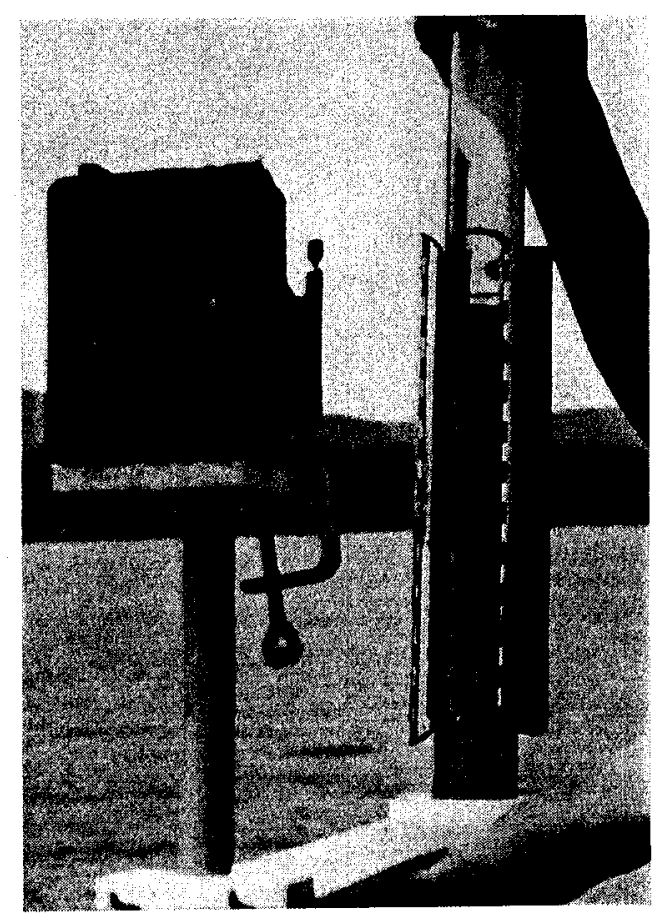

Abb. 3

Uferzone, wo das Schlammbild von Meter zu Meter wechselt und die Tätigkeit der Bodenfauna die Ausbildung horizontaler Schichtungen verhindert.

Das Aufschneiden der Schlammprobe in der Plexiglasröhre erforderte eine spezielle Bearbeitung derselben. Auf einer Länge von $30 \mathrm{~cm}$ des untern Rohrendes wurden gegenseitig achsenparallele Schlitze gefräst und davor ein ebenso langes Fenster ausgeschnitten. Letzteres wird längs einer Seite mit Klavierbandscharnier festgehalten, auf der andern Seite ist die eine Hälfte des Scharniers auf dem Fenster, die andere an der Leiste zwischen 
Schlitz und Fensterrand festgeschraubt. Das Fenster liegt gummigedichtet auf dem übrigen Plexiglasrohrteil auf und wird durch einen Stahldraht, welcher längs der beiden Scharnierhälften eingeführt wird, wasserdicht auf das Rohr gepresst. Die beiden seitlichen Schlitze werden weitgehend von aufgeleimten Gummibändern gegen Wasseraustritt gedichtet, insbesondere dann, wenn kein grosser Wasserdruck mehr auf den Bändern lastet. Die beiden I mm dicken Gummibänder sind in Rillen beidseitig der Schlitze aneinandergeschoben festgeleimt und können von einer Plexi-

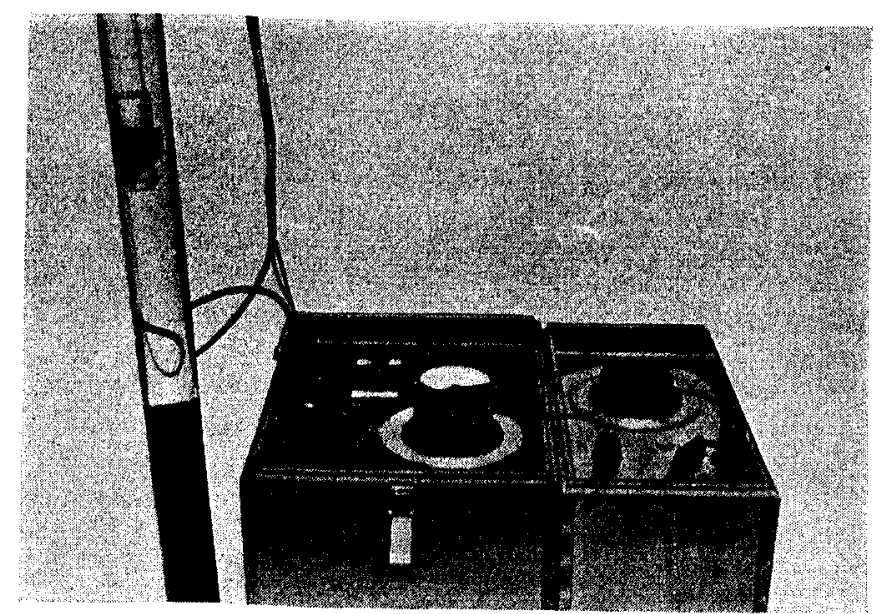

Abb. 4

glasscheibe, auf welcher die Millimeterskala eingeritzt ist, durchstossen werden. Vor dem Versenken der Apparatur werden die seitlichen Schlitze mit Leukoplast überzogen. Nach dem Hochheben der gefassten Probe entnimmt man zuerst die Wasserproben aus der den Schlamm überlagernden Wassersäule und löst erst nach Beendigung dieser Arbeiten die Leukoplastdichtungen ab. Erst jetzt stösst man der ganzen Länge nach die Plexiglasplatte durch den feinen Gummispalt ein, bis diese im gegenüberliegenden Spalt erscheint. Auf diese Weise verliert der lose Oberflächenschlamm kein Wasser und kann in seiner natürlichen Mächtigkeit photographiert werden.

Nach der photographischen Aufnahme werden zur mikroskopischen Analyse der Schlammzusammensetzung Proben entnommen und in Probegläschen von zirka $5 \mathrm{~mm}$ innerem Durchmesser aufbewahrt. Im Laboratorium wird der Inhalt dieser Gläschen mittels destilliertem Wasser ver-. 
dünnt und die Zahl der sedimentierten Organismen in der Kolkwitzschen Planktonkammer ermittelt.

Ferner gestattet der relativ grosse Innendurchmesser des Plexiglasrohrs (34 $\mathrm{mm})$ das Einführen einer Langstab-Glaselektrode mit Glasschutz, kombiniert mit einem eingeschmolzenen Silber-Silberchlorid-Halbelement und einer kleinen Leitfähigkeitszelle, wovon eine Elektrode gleichzeitig als Redoxelektrode Verwendung finden kann. Es gelingt so, sowohl $p H$,

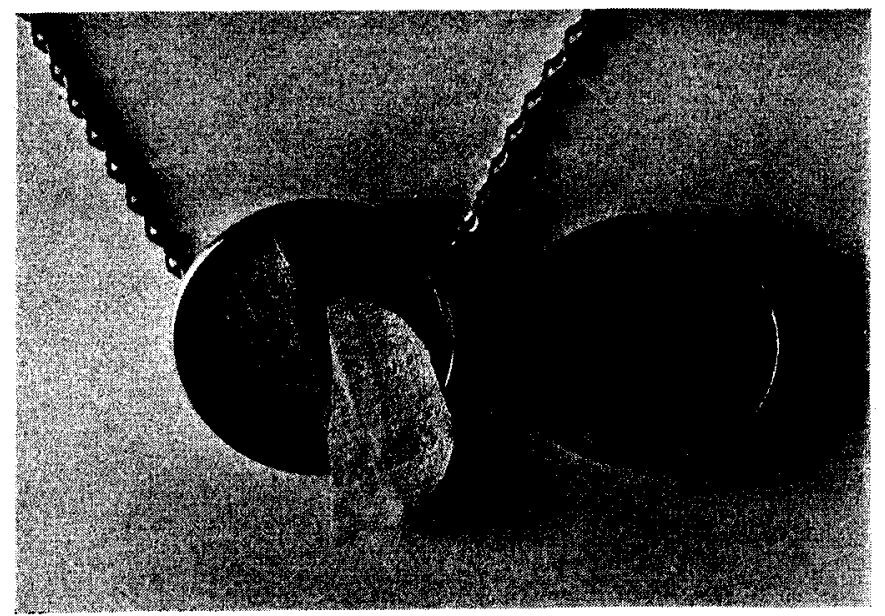

Abb. 5

Redoxpotential als auch die Leitfäbigkeit, welche oft unmittelbar über dem Schlamm ausgeprägt geschichtet sind, an Ort und Stelle zu ermitteln. Zur Verstärkung der zu messenden Ströme wird ein Batterie-pH-Gerät verwendet, welches in Zusammenarbeit mit $H$. JUCKER entwickelt wurde und gleichzeitig auch die Messung der Leitfähigkeit erlaubt. Das robust gebaute Gerät wiegt rund $5 \mathrm{~kg}$ und eignet sich besonders für Exkursionsuntersuchungen (Abb. 4).

Schliesslich verwenden wir das Lot noch dazu, den Zellulose- und Eimeissabbau auf dem Seeboden zu studieren. Richard [12] untersuchte den Zellulose- und Eiweissabbau in landwirtschaftlich genutzten Böden, wobei er die Zerreissfestigkeit der Testschnüre als Mass für den Abbau einführte. Indessen scheint uns dieses Mass insofern nicht ideal zu sein, als zu erwarten ist, dass eine Testschnur unregelmässig angegriffen wird und schliesslich die Menge abgebauten Materials von grossem Interesse ist. 
Ferner scheint es uns zweckmässig, die Abbaumenge pro Flächeneinheit angeben zu können, was wiederum bei Verwendung einer Testschnur auf Schwierigkeiten stösst.

Um die Geschwindigkeit des Abbaues messen zu können, gingen wir dazu über, je eine Kreishälfte Rohbaumwolle und Rohseide, welche vorher im Trockenschrank bei $80^{\circ} \mathrm{C}$ während $2 \mathrm{~h}$ erwärmt und noch weitere $2 \mathrm{~h}$ im Exsikkator getrocknet und schliesslich gewogen wurden, auf die Schlammoberfläche $\mathrm{zu}$ versenken. Als Halter dient ein kleines Rahmengestell (Abb. S), in welchem die Stoffplättchen zwischen feinen lackierten Metallsiebchen eingepresst werden. Als Verschluss dient ein Bajonettring. Das Rähmchen wird an zwei dünnen Kettchen auf die in einer Plexiglasröhre gefasste Schlammprobe gelegt und die Röhre mit Schlamm und überstehendem Wasser, gut verkorkt, in einem eisgekühlten Tragköcher transportiert. Die Röhren bleiben alsdann in der Dunkelheit in einem Raum von etwa $4^{\circ} \mathrm{C}$ während einiger Wochen aufbewahrt. Nach einer bestimmten Zeit werden die Rähmchen mit Inhalt herausgenommen, mit destilliertem Wasser gut gespült und im Trockenschrank und Exsikkator getrocknet. Die Menge des abgebauten Materials ist aus der Gewichtsdifferenz direkt ablesbar. Es scheint uns zweckmässig zu sein, den Eiweissbzw. Zelluloseabbau pro Quadratzentimeter und in 30 Tagen zum Beispiel anzugeben.

Zur Untersuchung der pH-, Redox- und Leitfähigkeitsverhältnisse sowie zur Ermittlung des Zellulose- und Eiweissabbaues verwenden wir unbearbeitete Plexiglasröhren.

Die Bearbeitung der Plexiglasröhre sowie die Anfertigung der übrigen Lotbestandteile wurde in Zusammenarbeit mit den Herren F. SCHIESSER und U. BRUGGER in der Konstruktionswerkstätte der Firma A. ZüLLIG, Apparatebau, Rheineck, ausgeführt.

\section{ZUSAMMENFASSUNG}

In der vorliegenden Arbeit wird ein neues Schlammprobenahmegerät beschrieben, das auf einfache und einwandfreie Weise gestattet, bodennahes Wasser und die jüngsten Schlammablagerungen zu erfassen. Dadurch wird die Möglichkeit geboten, den Chemismus der Sedimente, die Austauschvorgänge zwischen Schlamm und Wasser und die Biologie der Kontaktzonen zu studieren.

Ferner wird eine neue Methode zur Messung des Sedimentabsatzes be- 
schrieben, welche durch eine spezielle Bearbeitung der Schlammstechröhre aus Plexiglas ermöglicht wird.

Zur Messung der biologischen Aktivität der Ablagerungen in bezug auf Zellulose- und Eiweissabbau wird vorgeschlagen, halbkreisförmige Schnitzchen aus Rohbaumwolle (Zellulose) und Rohseide (Eiweiss) während einer bestimmten Zeit und Temperatur in einem Halter in Plexiglasröhren auf die Schlammoberfläche zu legen.

\section{SUMMARY}

A core sampling device is described in the preceding work. It allows to take out water lying just above the sediments as well as the youngest core layers. This gives the possibility to study the chemical properties of the sediments, the convertible process between sediment and water, and the biology of the zones of contact.

Furthermore, a new method is brought up to measure depositions of sediments with a special device of the core sampling apparatus made of Plexiglas.

The following method is proposed to measure the biological activity of the deposits with respect to the cellulose and protein degradation:

Slices of raw cotton (cellulose) and raw silk (protein) having the form of half a circle are placed in a cor.tainer which, in turn, is introduced in the Plexiglas tube above the surface of the sediments. The container remains there during a certain length of time and at a certain temperature.

\section{LITERATURVERZEICHNIS}

[I] Alsterberg, G., Die Dynamik des. Stoffpecbsels der Seen im Sommer (Lund 1935).

[2] Alsterberg, G., Die Winklersche Bestimmungsmetbode für in Wasser gelösten Sauerstoff sowie ibre Anwendung bei Anwesenbeit oxydierbarer Substanzen, Biochem. Z. 170 (1926).

[3] Hutchinson, G. E., Zitiert in: F. Ruttner, Grundriss der Limnologie (Berlin I952).

[4] JAAG, O., und ZülLIG, H., Vorläufge Mitteilungen über Beziebungen zmischen Cbemismus des Sedimentes und Trophiegrad eines Gemö̈ssers (im Druck).

[s] LiebmanN, H., Zur Biologie der Metbanbakterien, Ges.-Ing. 71, 1/4 (1950).

[6] MORTIMER, C. H., The Exchange of Dissolved Substances Between Mud and Water in Lakes, J. Ecol. 29 (I94I).

[7] NaumanN, E., Einfübrung in die Bodenkunde der Seen (Stuttgart 1930).

[8] OHLE, W., Die Bedeutung der Austauschborgänge zmiscben Scblamm und $W$ asser fïr den Stoffkreislauf der Gewässer, Vom Wasser 13 (1938).

[9] Perfiliew, B. W., Zur Mikrobiologie der Bodenablagerungen, Verh. int. Ver. theor. angew. Limnol. 4 (1929).

[10] VoN POST, H., Zitiert in [7].

[i ] FotoNí, H., Die rezenten Kaustobiolitbe und ibre Lagerstättcn, Abh. kgl. geol. Landesanstalt [N.F.] 55 (I908).

[12] RICHARD, F., Der biologiscbe Abbau pon Zellulose- und Eimeiss-Testscbnüren im Boden pon Wald und Rasengesellscbaften, Dissertation, ETH. Zürich (1945). 